\title{
Sinus Venarum
}

National Cancer Institute

\section{Source}

National Cancer Institute. Sinus Venarum. NCI Thesaurus. Code C34299.

A smooth-walled, systemic venous sinus, formed by a portion of the sinus venosus, located in the internal wall of the right atrium in the embryonic heart. 